\title{
Utilisation de sondes optiques miniatures en écoulement diphasique gaz-liquide Application à la mesure du taux de présence local et de la vitesse locale de la phase gazeuse
}

PAR

J.-P. Galaup

Laboratoire de Spectométrie Physique Grenoble
ET

\author{
J.-M. Delhaye \\ Centre d'Etudes Nucléaires de Grenoble \\ Service des Transferts Thermiques
}

\section{Introduction}

La vérification expérimentale de développements théoriques récents concernant les écoulements diphasiques, élaborés tant pour les applications au génie nucléaire qu'au génie chimique, nécessite une connaissance aussi fine que possible de la structure locale de tels écoulements. A cette fin, le développement de méthodes de mesure fiables revêt la plus grande importance.

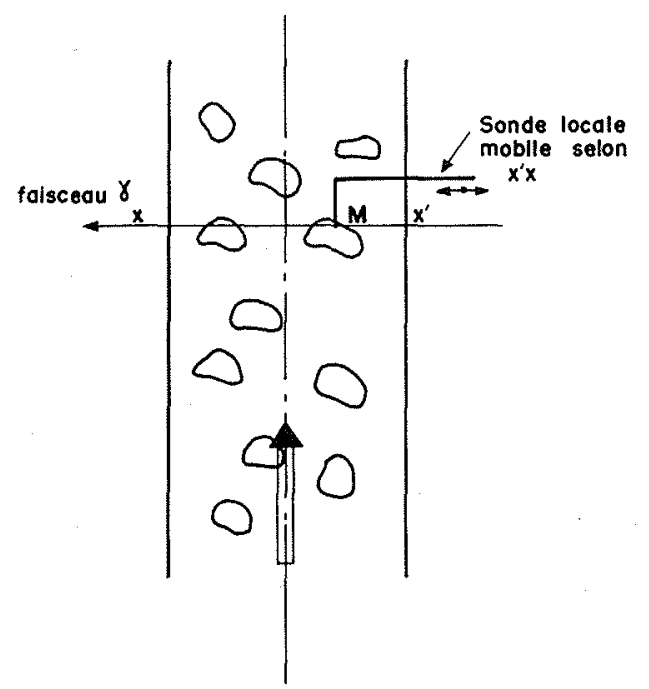

$1 /$ Schéma d'une expérience de qualification de sonde locale.
Dans cet article, des mesures locales réalisées par une sonde optique sont comparées à des mesures globales pour trois types différents de configuration d'écoulement : bulles, transition et bouchons.

$1^{\circ}$ La sonde optique mesure le taux de présence local du $\operatorname{gaz} \alpha(x)$ en différents points le long d'un diamètre $D$ d'un tube vertical dans lequel circule un écoulement eau-air ascendant. Les résultats sont comparés au taux de présence global moyen $\overline{R_{i}}$ mesuré le long du même diamètre par une méthode d'absorption de rayons $\gamma$ (fig. 1). L'identité suivante doit être satisfaite :

$$
\overline{R_{i}} \equiv 1 / D \int_{i}^{D} \alpha(x) d x \triangleq \varangle \alpha \ngtr
$$

$2^{\circ}$ A l'aide d'une sonde optique double, on peut mesurer la vitesse locale moyenne du gaz $\overline{V_{i}}$. Il existe alors une possibilité supplémentaire de comparaison avec le débit volumique du gaz $\overline{Q_{G}}$ mesuré par un débitmètre à turbine. Si l'écoulement est axisymétrique, on a en effet l'identité :

$$
\overline{Q_{r i}} \equiv \int_{11}^{H / 2} \alpha \overline{V_{r i}} 2 \pi r d r
$$

$3^{\circ}$ En outre, l'utilisation de sondes optiques doubles permet, sur la base d'un modèle statistique de l'écoulement, de déterminer la granulométrie et la surface spécifique d'un écoulement à bulles. Rappelons que la surface spécifique (ou volumique) $a$ est définie par :

$a=A / \vartheta=\frac{\sum \text { aires dinterface dans un volume } \mathcal{V}}{\text { volume } \vartheta}$ 


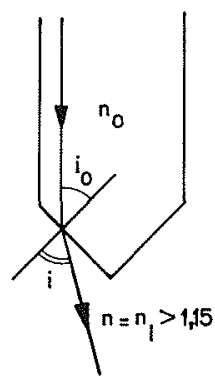

A - Réfraction

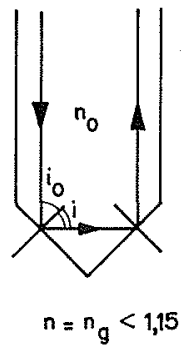

B_ Réflexion totale
$2 /$ Principe du détecteur optique de niveau.

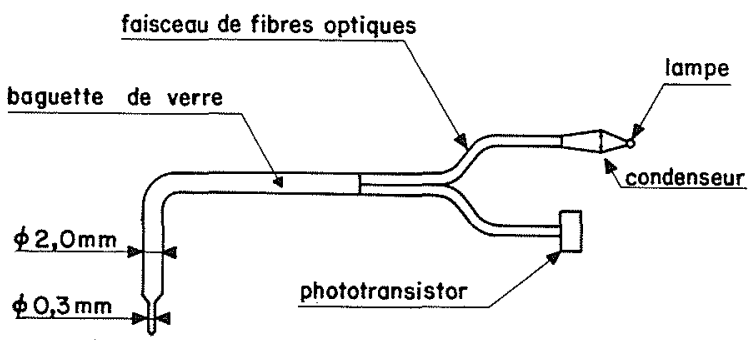

3/ Le système à tige de verre (Miller et Mitchie).

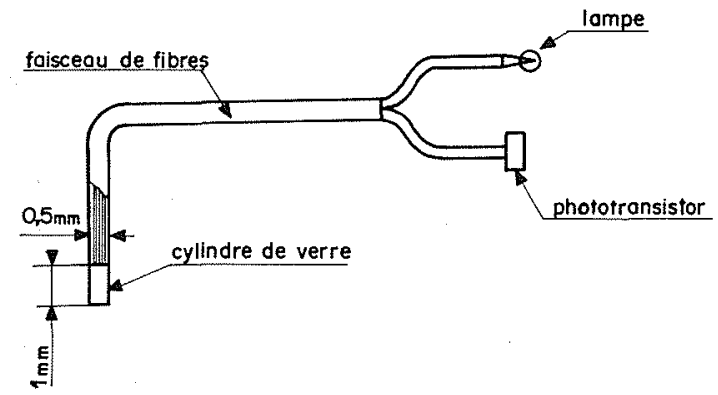

4/ Le système à faisceau de fibres (Hinata).

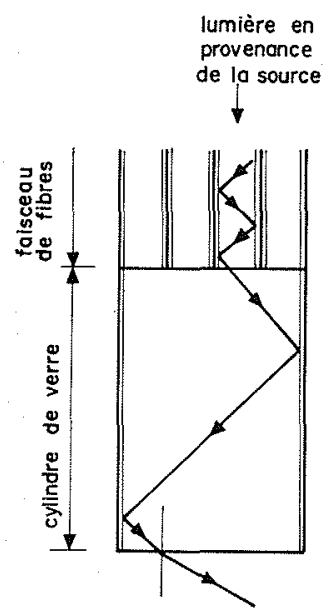

AIR

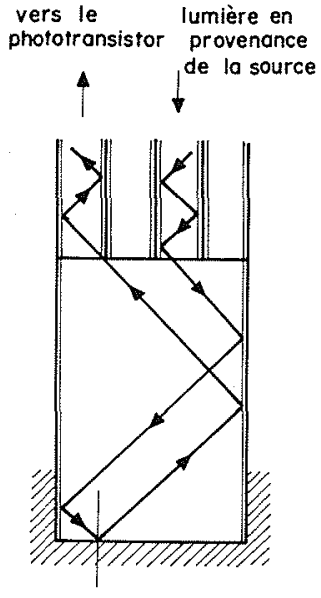

MERCURE
5/ Principe de la sonde optique de Hinata.

\section{2. Études antérieures sur l'utilisation de sondes optiques en écoulement diphasique}

A l'origine, le développement de la sonde optique a été dû à la nécessité d'étudier des écoulements de fréon-vapeur de fréon $\left(^{1}\right)$; on a pu en effet montrer [1] qu'une similitude existe entre des écoulements d'eauvapeur sous haute pression (140 bars) et de tels écoulements sous des pressions beaucoup plus faibles ( 23 bars au plus) et avec des puissances de chauffe moins importantes. La résistivité très élevée du fréon liquide ( $\simeq 10^{8} \Omega . \mathrm{cm}$ ) excluant l'emploi de sondes résistives et le recours aux sondes isocinétiques s'avérant délicat en raison de la méconnaissance des lois de prélèvement, il fallait trouver un dispositif nouveau.

La sonde optique utilise le phénomène de la réflexion totale déjà mis à profit dans les détecteurs de niveau dont nous rappellerons le principe. Dans une brève étude comparative, nous examinerons les réalisations de divers auteurs avant de nous attarder sur les sondes utilisées dans ce travail.

\subsection{Principe du détecteur optique de niveau.}

Considérons un prisme d'angle au sommet $90^{\circ}$, d'indice $n_{0}$ plongé dans un milieu d'indice $n$ (fig. 2). Un rayon lumineux arrivant sur l'une des faces du prisme sous un angle d'incidence $i_{0}$ sortira du prisme sous un angle d'émergence $i$ tel que :

$$
n \sin i=n_{0} \sin i_{0} \text { (loi de Descartes) }
$$

Il faut évidemment satisfaire l'exigence :

$$
\sin i=n_{0} / n \sin i_{0}<1
$$

d'où l'on déduit que, si le prisme est en verre $\left(n_{0}=1,62\right)$ et si $i_{0}=45$ degrés, il y aura réfraction uniquement lorsque $n$ sera supérieur à 1,15 et réflexion totale dans l'autre cas. Le dispositif décrit ci-dessus, utilisé à l'origine pour détecter la surface libre d'un liquide, sera aussi susceptible sous une forme miniaturisée de discerner la phase, liquide ou gaz, qui l'entoure pourvu que le mélange diphasique soit tel que :

$$
n_{r}<1,15<n_{J}
$$

Fondées sur ce principe, les sondes optiques seront ainsi utilisées avec succès dans les écoulements :

$$
\begin{aligned}
& \text { fréon-fréon vapeur } \ldots \ldots \ldots\left\{\begin{array}{l}
n_{L}=1,25 \\
n_{G}=1,02
\end{array}\right. \\
& \text { eau-vapeur ou eau-air } \ldots \ldots\left\{\begin{array}{l}
n_{L}=1,33 \\
n_{G}=1,00
\end{array}\right.
\end{aligned}
$$

\subsection{Réalisations antérieures}

Nous classerons les sondes réalisées en trois grandes classes selon le conducteur optique utilisé :

(I) Fréon 12, nom commercial du dichlorodifluorométhane $\mathrm{CF}_{2} \mathrm{Cl}_{2}$. 


\section{a) Le système à tige de verre}

Le modèle est la sonde réalisée par Miller et Mitchie $[2,3]$. Elle est constituée d'une baguette de verre étiré, de $2 \mathrm{~mm}$ de diamètre, coudée et polie à son extrémité sous la forme d'un cône d'angle au sommet 90 degrés, d'un conduit de lumière en $\mathrm{Y}$ dont les extrémités sont reliées à une lampe quartz-iodine $(6 \mathrm{~V}, 10 \mathrm{~W})$ d'une part et à un photo-transistor (Fairchild P 21) d'autre part (fig. 3). La pointe de la sonde a un diamètre compris entre 0,3 et $0,5 \mathrm{~mm}$. Notons que le conduit de lumière en $\mathrm{Y}$ est réalisé par un faisceau de fibres optiques ce qui assure une grande flexibilité. L'inconvénient majeur de ce type de sonde sont les pertes importantes qui obligent à l'emploi d'une lampe de forte puissance $(10 \mathrm{~W})$ donc grosse et ne permettant pas une miniaturisation du dispositif. Ces pertes se produisent essentiellement à la jonction entre le conduit de lumière et la baguette de verre, dans la baguette elle-même et par diffusion à l'extrémité à cause de la non-uniformité du poli.

Les auteurs ont testé leur sonde dans des écoulements eau-air pour lesquels ils assurent détecter des bulles de l'ordre de $0,5 \mathrm{~mm}$ de diamètre jusqu'à $3 \mathrm{~m} \cdot \mathrm{s}-1$ en vitesse apparente du liquide [2] et dans des écoulements fréon-fréon vapeur. Ils donnent dans [3] quelques résultats sur les profils de taux de présence du gaz obtenus. Un dispositif de vannes à fermeture rapide fournissait une valeur globale du taux de présence à laquelle étaient confrontées les mesures fournies par la sonde optique; malheureusement, la précision obtenue sur la valeur du taux de présence local était insuffisante pour que la comparaison puisse être valable.

Bell et al. [4] en 1972 et, plus récemment, Kennedy et Collier [5] ont utilisé la sonde de Miller et Mitchie pour étudier la structure de jets immergés dans un liquide. La partie sensible de la sonde a un diamètre de $0,36 \mathrm{~mm}$. Les signaux obtenus sont comparés à un seuil variable grâce à un circuit discriminateur. Ils indiquent pouvoir détecter des gouttes liquides d'un diamètre supérieur ou égal à $360 \mu \mathrm{m}$ mais ne précisent pas comment le niveau du seuil est ajusté. Selon Bell et al,, leur seuil était situé à mi-chemin entre les niveaux du signal dans le gaz (maximum) et dans le liquide (minimum), cependant, cette position n'est guère justifiée de façon convaincante.

\section{b) Le système à faisceau de fibres}

Mis au point par Hinata [6], ce dispositif a été utilisé dans des écoulements diphasiques mercure-air. Deux sondes de dimensions différentes ont été réalisées; l'élément de base qui les constitue est une fibre optique de $30 \mu \mathrm{m}(50 \mu \mathrm{m})$ de diamètre à double indice : le cœur de la fibre d'indice $n_{1}=1,62$ est entouré d'une gaine annulaire d'indice plus faible $n_{2}=1,52$. Avec cette disposition, les pertes lumineuses sont réduites. Une centaine environ de ces fibres sont liées ensemble de façon à former une sorte de grand Y (fig. 4). A l'extrémité du faisceau de fibres qui atteint alors $0,5 \mathrm{~mm}(0,7 \mathrm{~mm})$ de diamètre, on a collé un petit cylindre de verre à double indice également, de même diamètre et de $1 \mathrm{~mm}$ de long. La partie sensible de la sonde est aplanie et polie. Les deux autres extrémités du faisceau de fibres sont respectivement connectées à une source lumineuse (lampe de projection munie d'un système focalisant) et à un phototransistor dont les caractéristiques ne sont pas spécifiées.

Cette sonde ne fonctionne pas selon le même schéma que le détecteur de niveau mais bien plutôt comme un détecteur de proximité, tel celui que décrit Powell [7]. Ce système utilise la réflexion de la lumière à l'extrémité plane de la partie sensible, sur laquelle le mercure joue le rôle d'un petit miroir (fig. 5). Une conséquence de ce mode de fonctionnement est que le temps de réponse de la sonde, lors de la percée de la bulle, est plus court que celui observé lors de la pénétration dans le liquide, ce qui est contraire à l'observation générale. Cela s'explique cependant aisément par le fait que la zone sensible «voit " la surface réfléchissante de l'interface air-mercure arriver sur elle avant qu'elle ne la touche vraiment.

$\mathrm{Ce}$ fonctionnement introduit donc une erreur supplémentaire qui, vu l'ordre de grandeur des temps de réponse ( 3 et $6 \mathrm{~ms}$ pour la sonde de $0,5 \mathrm{~mm}$, 5 et $8 \mathrm{~ms}$ pour celle de $0,7 \mathrm{~mm}$ ) ne semble pas négligeable.

\section{c) Le système à fibre unique pliée en $U$}

Ce système imaginé par Danel et Delhaye $[8,9]$ est la première forme miniaturisée de la sonde optique. Dans sa première version, elle était constituée par (fig. 6) :

- une lampe à incandescence miniature de faible puissance (300 $\mathrm{mW}$ environ);

- une fibre optique à double indice de $40 \mu \mathrm{m}$ de diamètre ;

- un photodarlington BPX 30 choisi pour sa grande sensibilité (minimum : $100 \mathrm{~mA} \cdot \mathrm{mW}^{-1} \cdot \mathrm{cm}^{-2}$ ).

L'extrémité de la sonde est obtenue en pliant la fibre à $180^{\circ}$ par un procédé technologique spécial; cela permet d'atteindre un diamètre de la partie sensible de l'ordre de $100 \mu \mathrm{m}$. La fibre est placée à l'intérieur d'un tube en acier inoxydable de $2 \mathrm{~mm}$ de diamètre extérieur qui assure la rigidité de l'ensemble. Cette sonde a fait l'objet d'essais en fréon-vapeur de fréon $[10,11,12]$.

\section{Sonde optique utilisée dans cette étude}

C'est une version améliorée de la sonde Danel et Delhaye (fig. 7).

La partie sensible de la sonde émerge d'un minitube de $0,3 \mathrm{~mm}$ de diamètre extérieur, et le récepteur a été

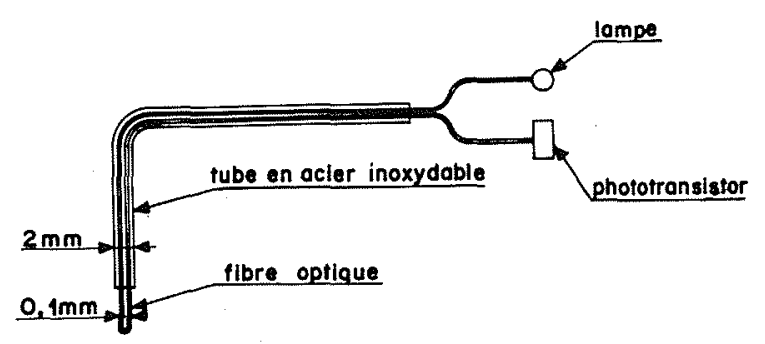

6) Le système à fibre unique en $U$ (Danel et Delhaye). 


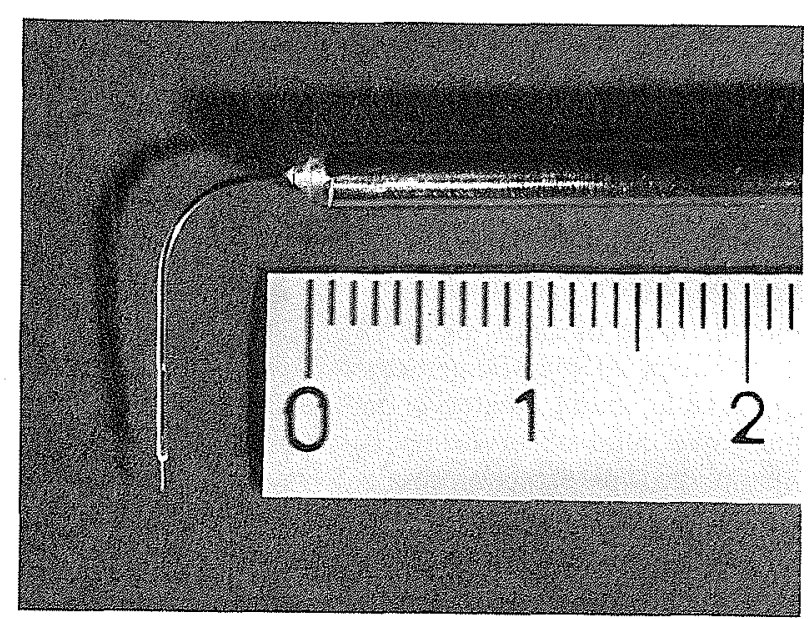

$7 /$ La sonde optique miniature simple.
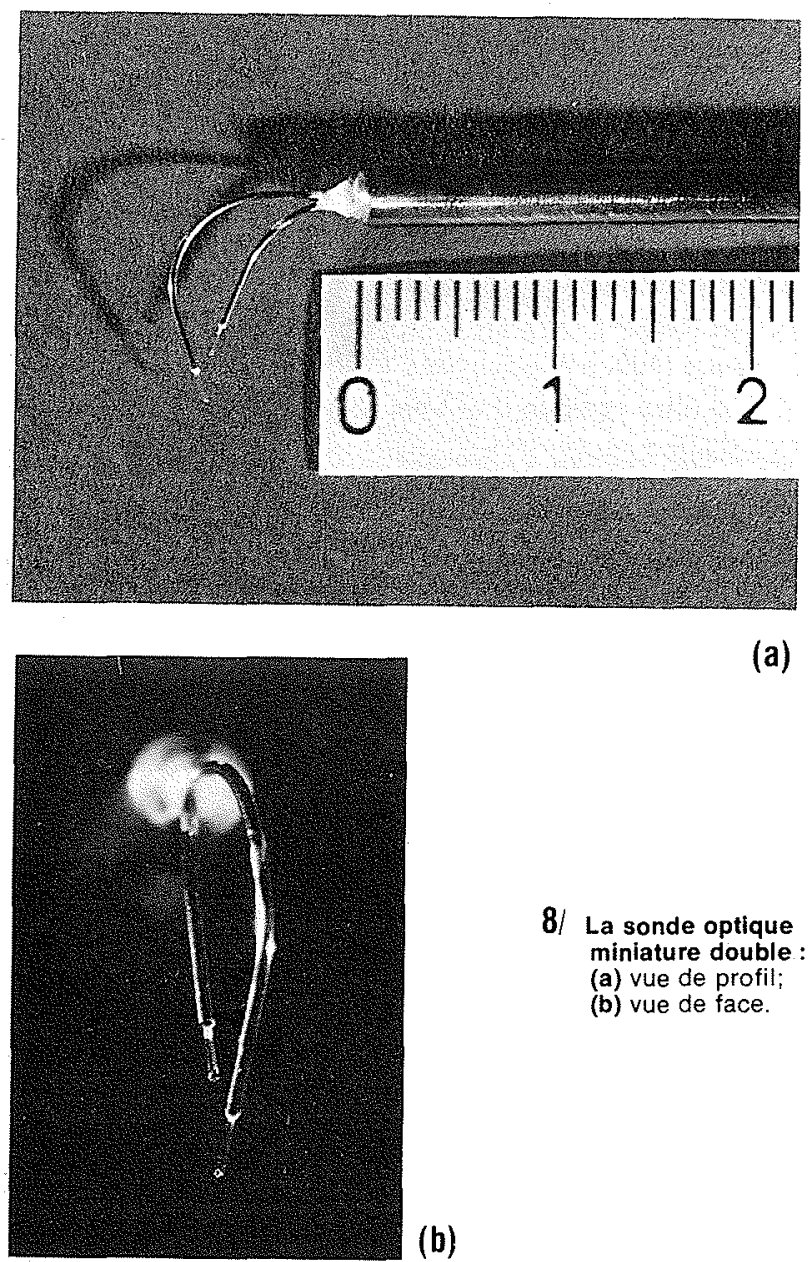

(a)
8/ La sonde optique miniature double: (a) vue de profil; (b) vue de face. (b)

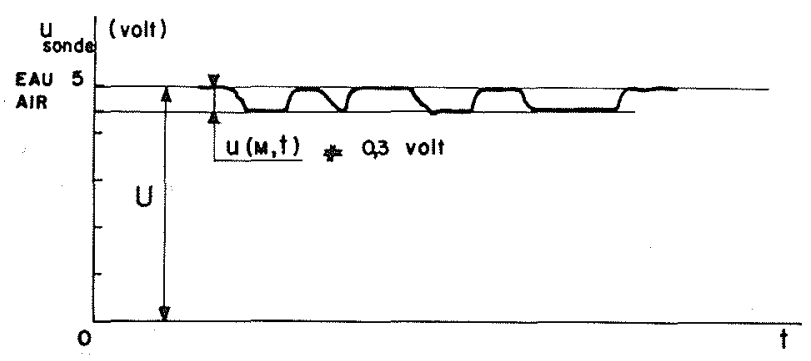

9/ Signal prélevé à la sortie de la sonde optique. changé : on utilise un phototransistor TIL 81 de bande passante élevée $(200 \mathrm{kHz}$ contre $20 \mathrm{kHz}$ pour le BPX 30) associé à un amplificateur intégré dans le corps de la sonde [13].

Pour les mesures de la vitesse des interfaces, on a utilisé une bisonde optique (fig. 8-a et 8-b). Les deux extrémités sensibles sont distantes de $2,3 \mathrm{~mm}$. Le tableau 1 permet de comparer les caractéristiques et performances des principaux types de sondes optiques mises au point à ce jour.

\section{Chaîne de mesure et traitement du signal}

\subsection{Mesure du taux de présence local}

Le signal électrique délivré par la sonde optique contient une composante continue de l'ordre de $5 \mathrm{~V}$ superposée au signal utile de $300 \mathrm{mV}$ d'amplitude, témoignant du passage des bulles (fig. 9). La composante continue est éliminée à l'aide d'un circuit analogique détecteur de valeur crête. Un circuit discriminateur à seuil ajustable assure l'obtention d'un signal binaire indicateur de la phase présente autour du point de mesure. Ce signal, moyenné analogiquement, donne le taux de présence local du gaz. On peut également en extraire la fréquence moyenne d'arrivée des bulles (fig. 10).

\subsection{Mesure de la vitesse locale du gaz}

On utilise une bisonde optique dont les deux extrémités sensibles séparées par une distance $d$ sont situées sur une droite parallèle à l'axe de la conduite.

Si $\theta(k)$ désigne le temps de transit de l'interface d'une bulle $k$ entre les sondes, la vitesse locale de la bulle $k$ sera :

$$
V_{t_{i}}(k)=d / \theta(k)
$$

et donc la vitesse locale du gaz pendant le temps de mesure $T$ s'écrira :

$$
\overline{V_{i}}=1 / N(T) \sum_{k=1}^{x|T|} V_{G}(k)
$$

où $N(T)$ est le nombre de bulles passées entre les deux sondes pendant $T$.

Expérimentalement, on peut apprécier la vitesse locale du gaz en utilisant soit la distribution des intervalles de temps de transit (fig. 11), soit la fonction d'intercorrélation (fig. 12).

Dans sa thèse [14], l'un d'entre nous a utilisé ces deux méthodes et montré, qu'à la précision obtenue, les résultats obtenus par ces deux procédés sont en moyenne équivalents. En principe cependant, la distribution des temps de transit fournit plutôt une vitesse moyenne et la fonction d'intercorrélation, une vitesse intermédiaire entre la vitesse moyenne et la vitesse la plus probable. 


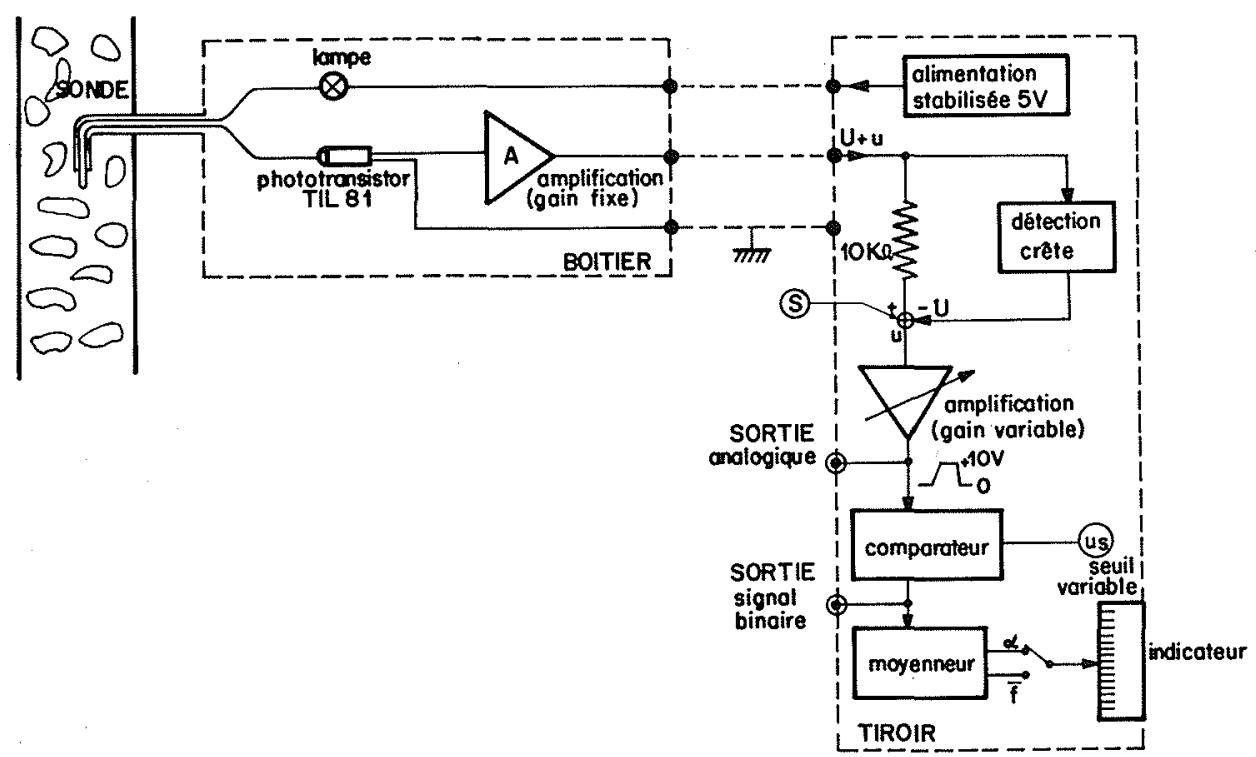

$10 /$ Schéma synoptique du traitement du signal de la sonde optique.

\begin{tabular}{|c|c|c|c|c|c|c|c|c|c|}
\hline $\begin{array}{c}\text { TYPE } \\
\text { DE SONDE }\end{array}$ & EMETTEUR & $\begin{array}{l}\text { CONDUCTEUR } \\
\text { OPTIQUE }\end{array}$ & RÉCEPTEUR & $\begin{array}{l}l_{m} \\
\text { élect. } \\
(\mu s)\end{array}$ & $\begin{array}{l}t_{t \prime} \\
\text { élect. } \\
\text { (ụs) }\end{array}$ & FLUIDE & $\begin{array}{l}V_{\text {moswnmu }} \\
\left(\mathrm{m}^{\prime} \cdot \mathrm{s}^{-1}\right)\end{array}$ & $\mid \begin{array}{c}t_{n} \\
\text { mesuré } \\
(\mu \mathrm{s})\end{array}$ & $\begin{array}{c}t_{d} \\
\text { mesuré } \\
(\mu \mathrm{s})\end{array}$ \\
\hline \begin{tabular}{|c|} 
MILLER \\
et MITCHIE \\
{$[2,3]$} \\
1969
\end{tabular} & $\begin{array}{l}\text { lampe } \\
\text { quartz-iode } \\
6 \mathrm{~V}, 10 \mathrm{~W} \\
+ \text { lentille } \\
\text { collectrice }\end{array}$ & $\begin{array}{l}\text { baguette de verre } \\
\varnothing 2 \mathrm{~mm} \\
\text { usinée en cône } \\
\text { à son extrémité } \\
\varnothing 0,3 \mathrm{~mm}\end{array}$ & $\begin{array}{c}\text { phototransistor } \\
\text { P } 21 \text { (SGS-ATES) } \\
\text { sensibilité } \\
\text { très faible } \\
20 \mu \mathrm{A} \cdot \mathrm{mW}^{-1} \cdot \mathrm{cm}^{-2} \\
\text { (valeur min.) }\end{array}$ & $\simeq 50$ & $\simeq 50$ & $\begin{array}{l}\text { fréon- } \\
\text { fréon } \\
\text { vapeur }\end{array}$ & 1 à 3 & $\begin{array}{c}150 \\
\grave{a} \\
350\end{array}$ & $\begin{array}{l}40 \\
\grave{a} \\
50\end{array}$ \\
\hline $\begin{array}{c}\text { HINaTA } \\
{[6]} \\
1972\end{array}$ & $\begin{array}{c}\text { lampe } \\
+ \text { - projecteur }\end{array}$ & $\begin{array}{l}\text { faisceau de } \\
\text { fibres optiques } \\
+ \text { baguette } \\
\text { de verre } \\
\varnothing 0,5 \mathrm{~mm}\end{array}$ & - phototransistor & $?$ & $?$ & $\begin{array}{c}\text { mercure- } \\
\text { air }\end{array}$ & $\begin{array}{l}\text { quelques } \\
\mathrm{cm} \cdot \mathrm{s}^{-1}\end{array}$ & 3000 & 6000 \\
\hline $\begin{array}{c}\text { Danel } \\
\text { et DelHaye } \\
{[8,9,11]} \\
1971\end{array}$ & $\begin{array}{c}\text { lampe } \\
\text { miniature } \\
\text { à filament } \\
4,5 \mathrm{~V}, 60 \mathrm{~mA}\end{array}$ & $\begin{array}{c}\text { fibre optique } \\
\varnothing 40 \mu \mathrm{m} \\
\text { pliée à } 180^{\circ} \\
\varnothing 0,1 \mathrm{~mm}\end{array}$ & 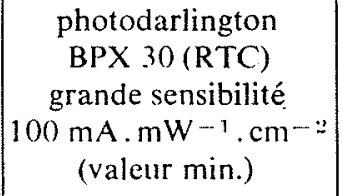 & $\begin{array}{c}60 \\
\text { (valeur } \\
\text { typ.) }\end{array}$ & $\begin{array}{c}40 \\
\text { (valeur } \\
\text { typ.) }\end{array}$ & $\begin{array}{l}\text { fréon- } \\
\text { fréon } \\
\text { vapeur }\end{array}$ & 0,5 & $\begin{array}{c}200 \\
\grave{a} \\
300\end{array}$ & $\begin{array}{c}80 \\
\grave{a} \\
120\end{array}$ \\
\hline $\begin{array}{l}\text { A.I.D. } \\
1974\end{array}$ & $\begin{array}{c}\text { lampe } \\
\text { miniature } \\
\text { à filament } \\
4,5 \mathrm{~V}, 60 \mathrm{~mA}\end{array}$ & $\begin{array}{c}\text { fibre optique } \\
\varnothing 40 \mu \mathrm{m} \\
\text { pliée à } 180^{\circ} \\
\varnothing 0.1 \mathrm{~mm}\end{array}$ & $\begin{array}{c}\text { phototransistor } \\
\text { TIL } 81 \\
\text { (Texas Inst.) } \\
\text { sensibilité faible } \\
1 \mathrm{~mA} \cdot \mathrm{mW}^{-1} \mathrm{~cm}^{-2} \\
\text { (valeur min.) }\end{array}$ & $\begin{array}{c}8 \\
\text { (valcur } \\
\text { typ.) }\end{array}$ & $\begin{array}{c}6 \\
\text { (valeur } \\
\text { typ.) }\end{array}$ & eau-air & 1 & 80 & 40 \\
\hline
\end{tabular}




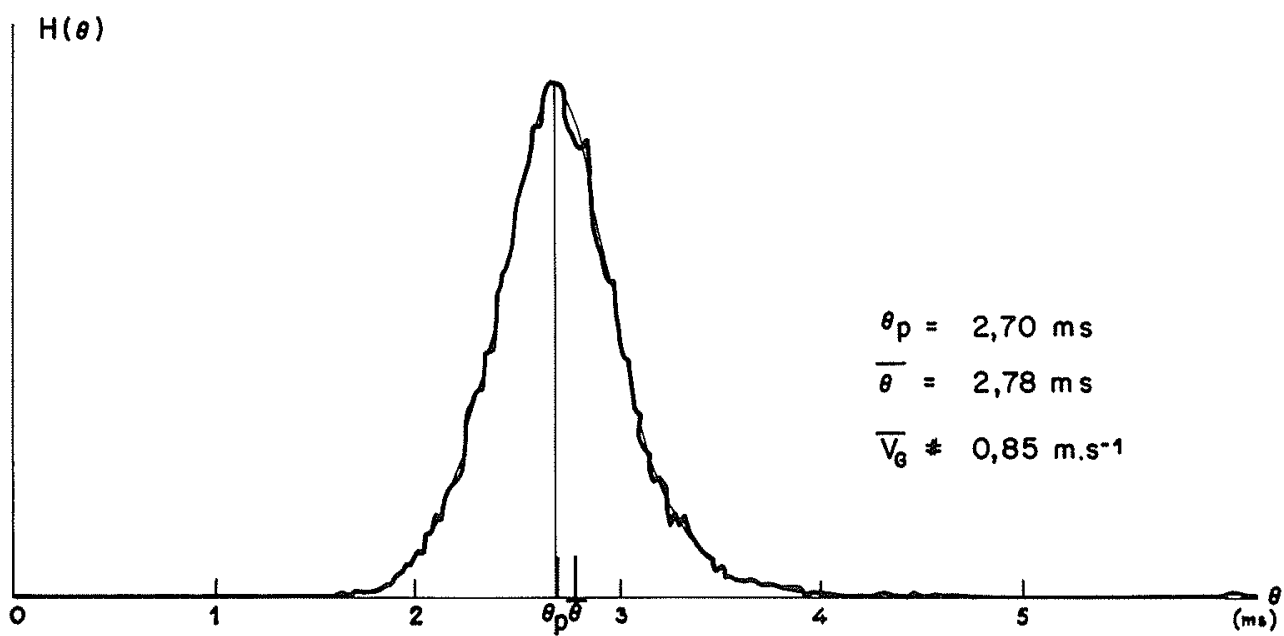

$$
\begin{aligned}
\text { ESSAI } 0-1: \bar{j}_{L}=0,51 \mathrm{~m} \cdot \mathrm{s}^{-1}, x=2,0 \cdot 10^{-4} \\
\text { bisonde optique }(d=2,3 \mathrm{~mm}) \text { au centre du canal }
\end{aligned}
$$

11/ Histogramme des temps de transit des bulles.

\section{Dispositif expérimental}

\subsection{L'installation d'essais (fig. 13)}

Dans une conduite verticale, on réalise un écoulement diphasique eau-air par injection d'air à la base.

Une pompe centrifuge, susceptible de fournir un débit de $20 \mathrm{~m}^{3} \mathrm{~h}^{-1}$ avec une hauteur manométrique de $40 \mathrm{~m}$ alimente en eau un réservoir mis en pression par de l'air à sa partie supérieure. Le débit d'eau est réglé par la pression du réservoir et par une vanne à pointeau. Un ensemble de chauffage permet de réguler la température de l'eau entre la température ambiante et $100^{\circ} \mathrm{C}$ : il comporte deux thermoplongeurs de puissance unitaire $6 \mathrm{~kW}$ situés dans le réservoir et une préchauffe de $8 \mathrm{~kW}$ placée à l'amont de la section de mesure. Les trois thermoplongeurs sont couplés à un indicateur-régulateur de température Pyrectron piloté par un thermomètre à résistance de platine placé dans un doigt de gant à l'entrée du canal d'essais. Au bas de la partie verticale du circuit d'essais, l'air en provenance d'une batterie de bouteilles est injecté au travers d'une série de 96 trous d'un diamètre égal à $0,5 \mathrm{~mm}$ et disposés en quinconce

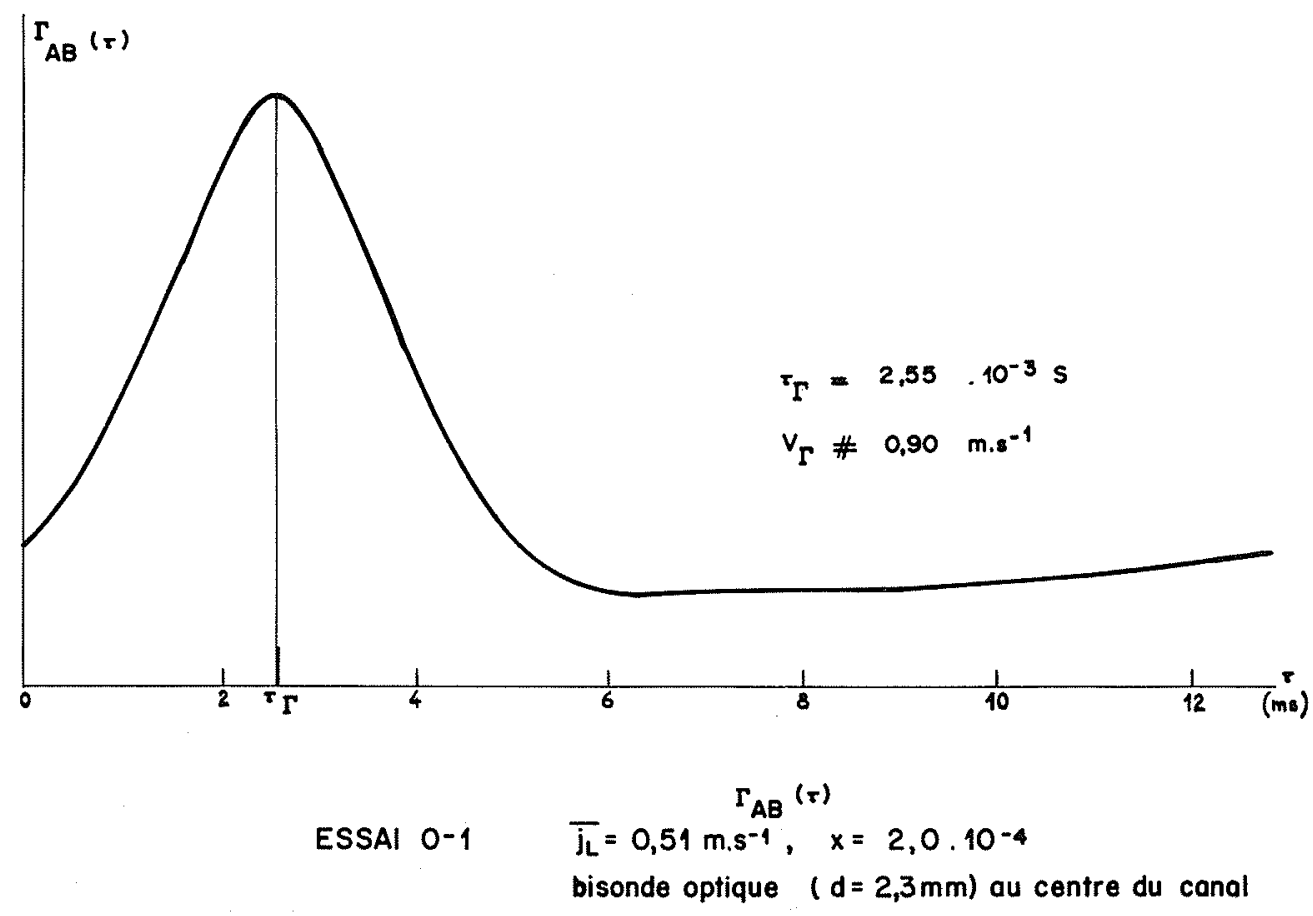




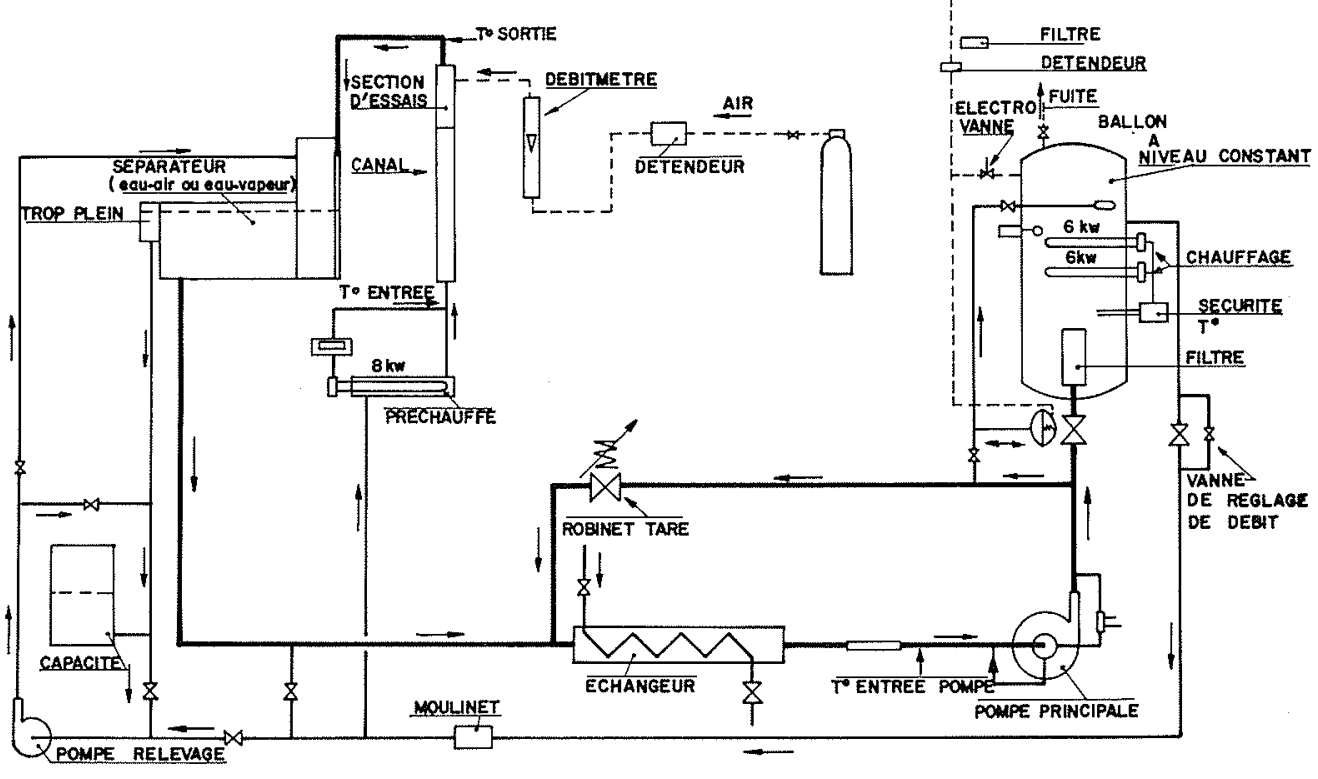

13/ Schéma de l'installation d'essais.

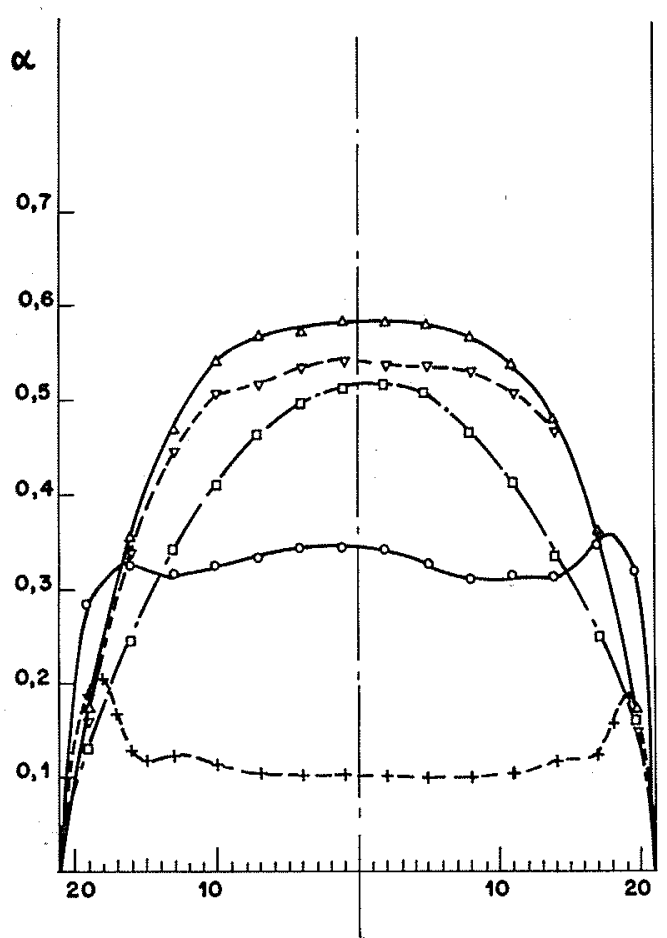

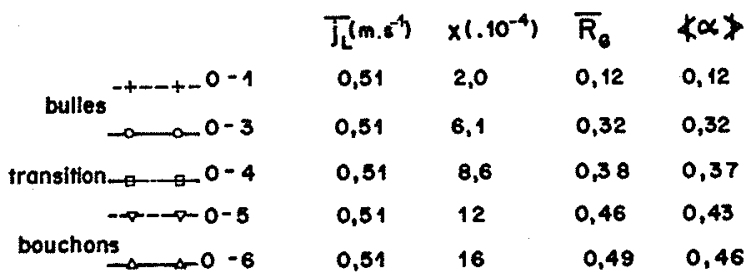

14/ Profils du taux de présence local (sonde optique).

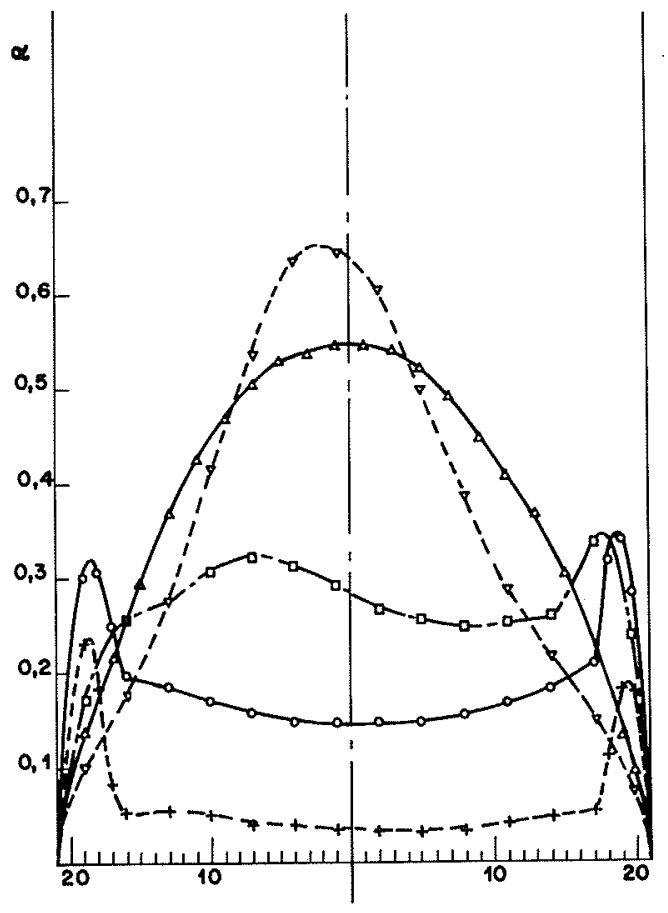

$\bar{J}_{L}\left(\mathrm{~m} . \mathrm{s}^{-4}\right) \times\left(.10^{-4}\right) \overline{R_{8}} \quad \nless \alpha \ngtr$

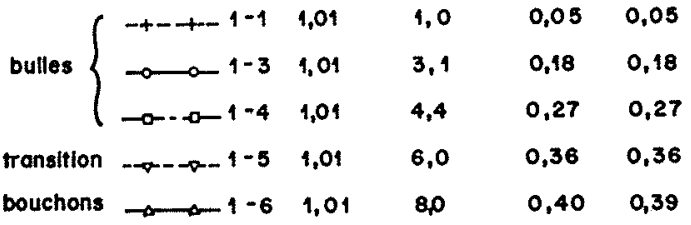

15/ Profils du taux de présence local (sonde optique). 

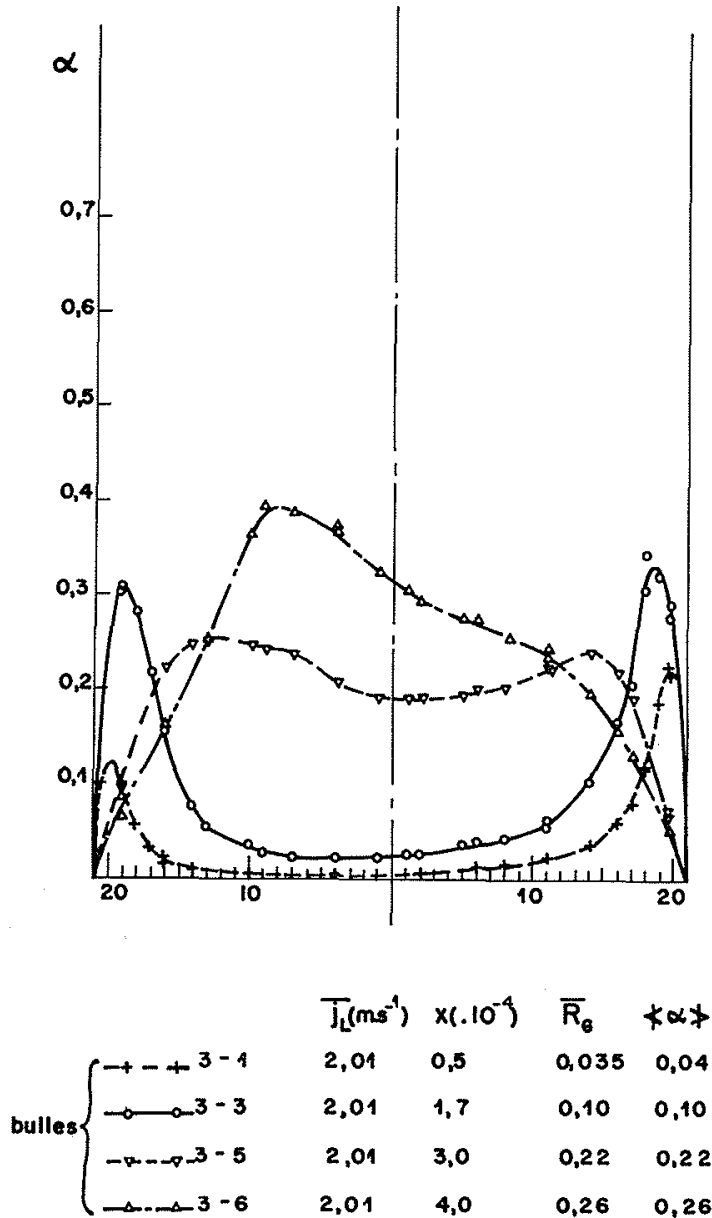

16/ Profils du taux de présence local (sonde optique).

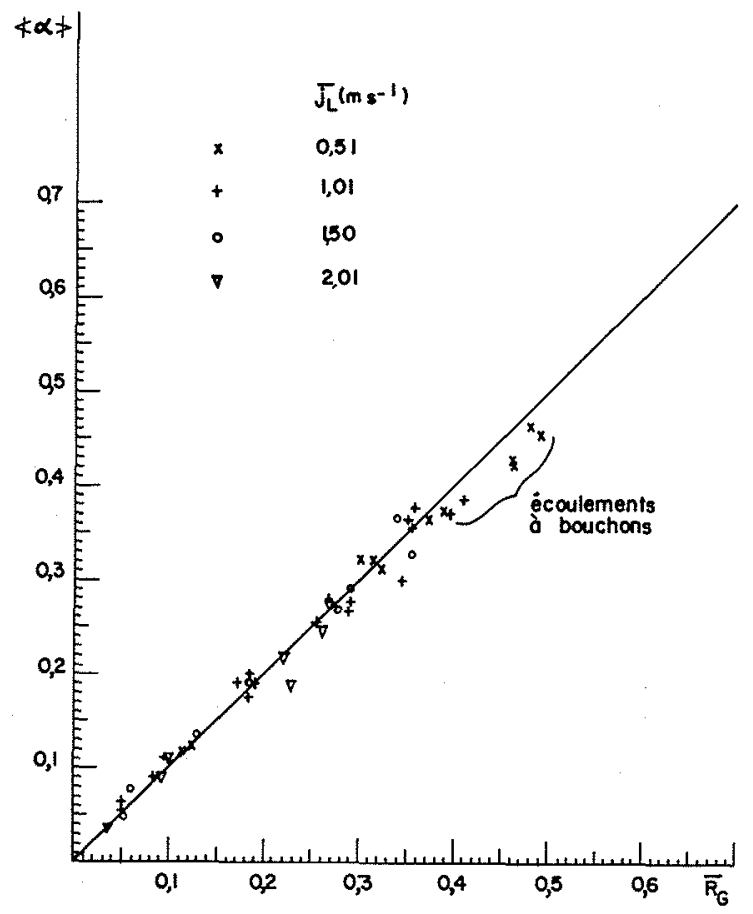

17/ Comparaison sonde optique et absorption . sur quatre rangées. A la sortie du canal d'essais, le mélange eau-air débouche à gueule bée dans une cuve de tranquillisation. Les bulles d'air s'échappent à l'atmosphère et l'eau seule retourne à la pompe en passant par un échangeur de chaleur. Les températures d'entrée et de sortie du canal d'essais, ainsi que la température d'entrée de la pompe, sont mesurées à l'aide de thermocouples chromel-alumel placés dans des doigts de gant. Les soudures froides sont plongées dans des enceintes régulées automatiquement à $0 \pm 0,02{ }^{\circ} \mathrm{C}$, type Zérostable. Les variations de température au cours du temps sont relevées sur un enregistreur potentiométrique Meci.

Le débit d'eau $\overline{Q_{L}}$ est mesuré à l'aide d'un débitmètre à turbine Faure-Herman T 16. L'erreur relative totale sur le débit liquide est estimée à $1 \%$ environ [15].

La mesure du débit d'air $\overline{Q_{G}}$ utilise un débitmètre à flotteur Rota G 1 250-3 000 associé à un thermomètre et à un manomètre Bourdon.

\subsection{Le banc de gammagraphie}

Le banc de gammagraphie utilise une source $\gamma$ d'Américium $\mathrm{Am}_{45}^{24}$ dont la période de demi-vie est égale à 458 ans. L'activité contenue de la source est de l'ordre de $1 \mathrm{Ci}$. Cette valeur ne tient pas compte de l'autoabsorption de la source, ni de l'absorption due aux enveloppes de protection.

Le pic d'émission principal des photons $\gamma$ est de basse énergie (environ $60 \mathrm{keV}$ ).

Le photomultiplicateur (P.M.) utilise un cristal d'iodure de sodium comme scintillateur. Il est relié à une chaîne de comptage classique comprenant, outre l'alimentation haute-tension du P.M., un tiroir de mise en forme des impulsions, un sélecteur monocanal d'amplitude, un intégrateur analogique servant au contrôle et un compteur à six décades. La sortie des résultats s'effectue sur une imprimante.

L'ensemble, source, P.M. et collimateurs, est monté sur un bras support rigide, lui-même soudé à un chariot susceptible de se mouvoir selon deux directions orthogonales:

- axe $Z$ vertical,

- axe $Y$ horizontal,

- l'axe $X$ étant l'axe du faisceau $\Upsilon$.

Les déplacements sont assurés par des moteurs pas à pas commandés par une chaîne électronique permettant de programmer le nombre et la longueur des pas selon les deux axes indiqués. Le plus petit deplacement permis est de $0,5 \mathrm{~mm}$ selon $Z$ et 0,05 selon $Y$. La distance source-P.M. est d'environ $40 \mathrm{~cm}$.

\section{Résultats obtenus}

Les mesures ont porté sur un écoulement ascendant eau-air, pour trois types de configurations : bulles, transition et bouchons. Le titre de l'écoulement varie entre $0.5 \cdot 10^{-4}$ et $16 \cdot 10^{-4}$ et la vitesse apparente du liquide entre 0,5 et $2 \mathrm{~m} . \mathrm{s}^{-1}$. 


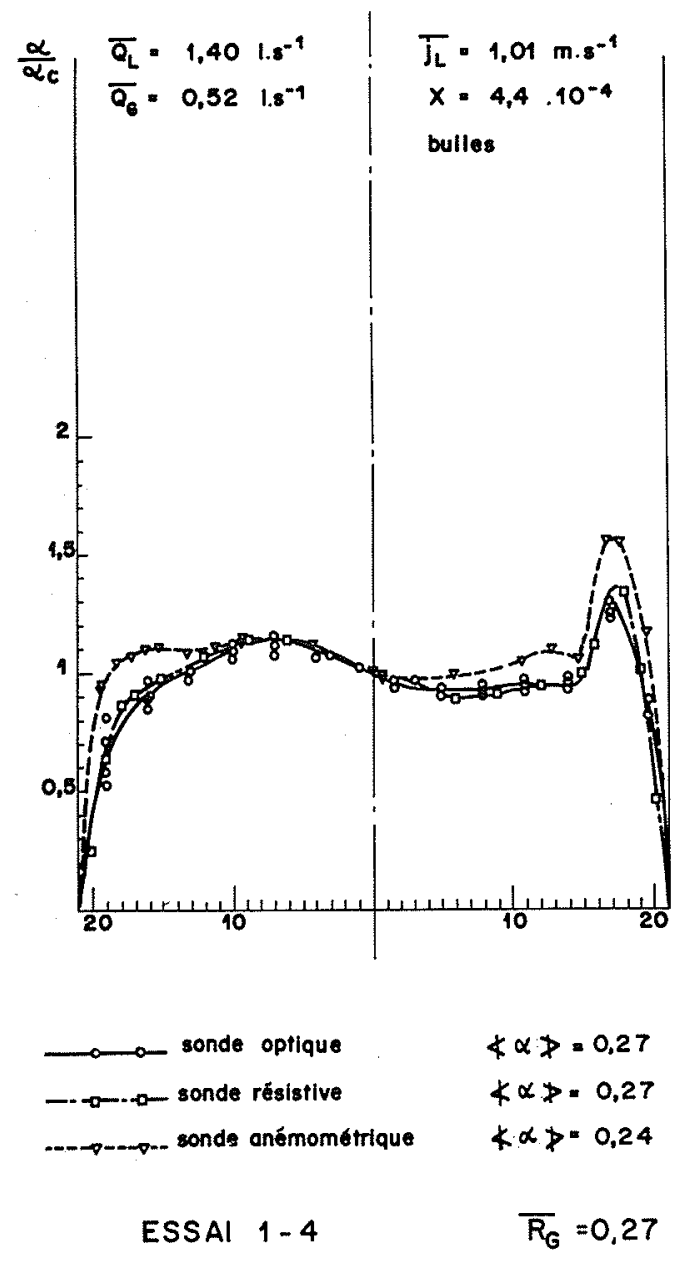

18/ Comparaison des sondes locales.

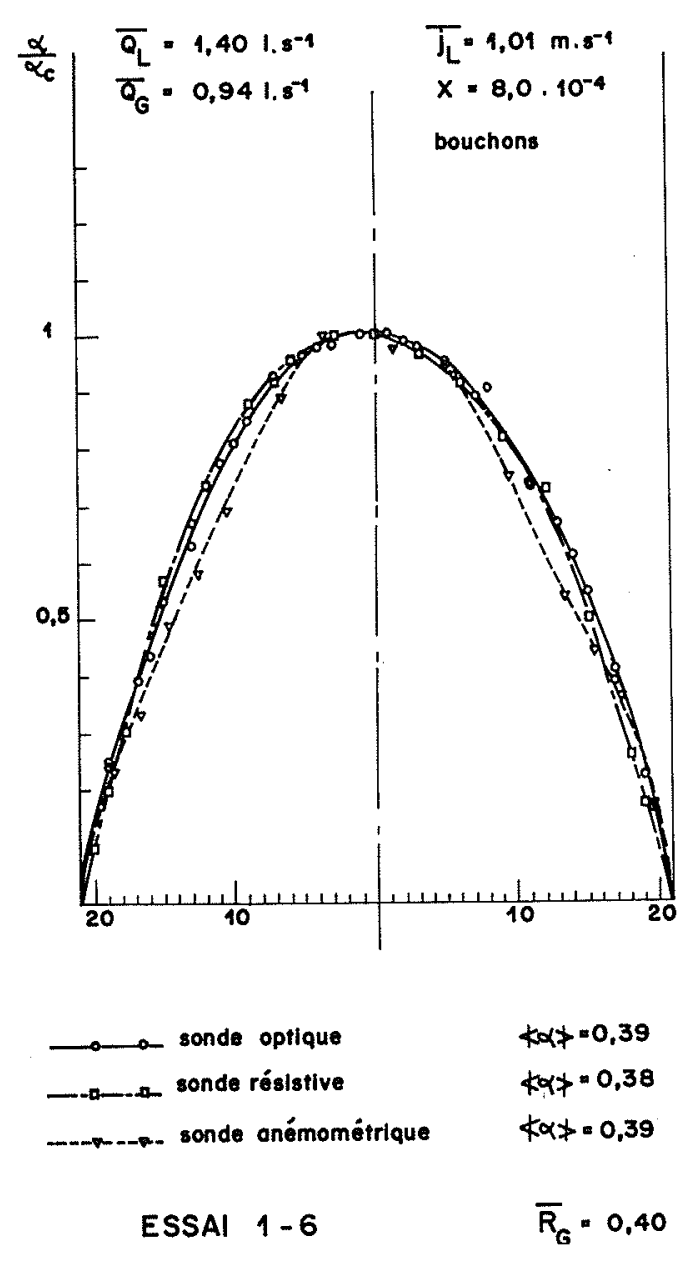

19/ Comparaison des sondes locales.

\subsection{Profils de taux de présence}

\section{a) Comparaison avec l'absorption $\gamma$}

Les figures 14 à 16 montrent l'évolution des profils de taux de présence, pour une vitesse apparente de liquide donnée, lorsque le titre massique du gaz varie.

Notre étude montre que :

- l'influence de la position du seuil du circuit discriminateur est faible: le taux de présence moyenné sur le diamètre $\$ \alpha \ngtr$ varie seulement de $3 \%$ lorsque la hauteur du seuil varie de $70 \%$ autour d'une position médiane ;

- un bon accord entre $\Varangle \alpha \ngtr$ et $\overline{R_{G}}$ est obtenu en plaçant le seuil à environ $40 \%$ de l'amplitude maximale du signal.

Les résultats de la corrélation entre le taux de présence global $\overline{R_{i}}$ mesuré par l'absorption gamma el $\nless \alpha \neq$ sont donnés figure 17 .

\section{b) Comparaisons avec d'autres sondes}

Dans le même canal d'essais et pour les mêmes conditions d'écoulement (pression, vitesse appartente du liquide. titre) nous avons comparé les résultats précédents avec les mesures fournies par :
- une sonde résistive miniature (diamètre de la partie sensible $20 \mu \mathrm{m}$ et diamètre du tube immergé $0,2 \mathrm{~mm}$ ) [14]:

- une sonde anémométrique à film chaud Disa, type $55 \mathrm{R} 42$ (diamètre de la partie sensible $0,3 \mathrm{~mm}$ et du tube immergé $5 \mathrm{~mm}$ ) $[14,16]$.

Quelques résultats sont présentés sur les figures 18 à 21. On notera que bien que les essais avec les différentes sondes aient été effectués avec plusieurs mois d'intervalle, les profils obtenus par chacune d'elles redonnent les mêmes dissymétries. D'autre part, les dimensions importantes de la sonde anémométrique ne semblent pas avoir d'influence sur les résultats obtenus.

\subsection{Profils de la vitesse locale du gaz}

Lal figure 22 donne les comparaisons expérimentales entle les différentes vitesses du gaz mesurées. On notera que la vitesse moyenne est systématiquement supérieure à la vitesse la plus probable, résultat en accord avec ceux rapportés par Serizawa [17].

Les vitesses mesurées par corrélation semblent, compte tenu de la précision des mesures, intermédiaires entre la vitesse moyenne et la vitesse la plus probable. Nos données confirment cependant les observations de 


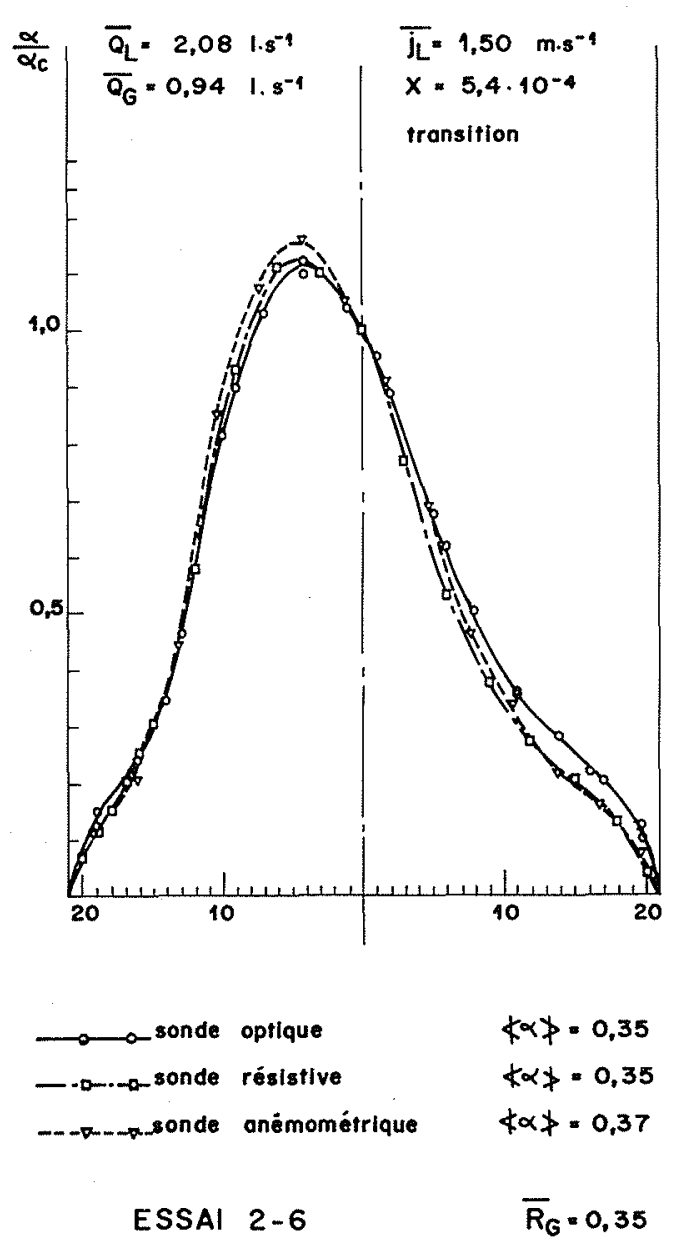

20/ Comparaison des sondes locales

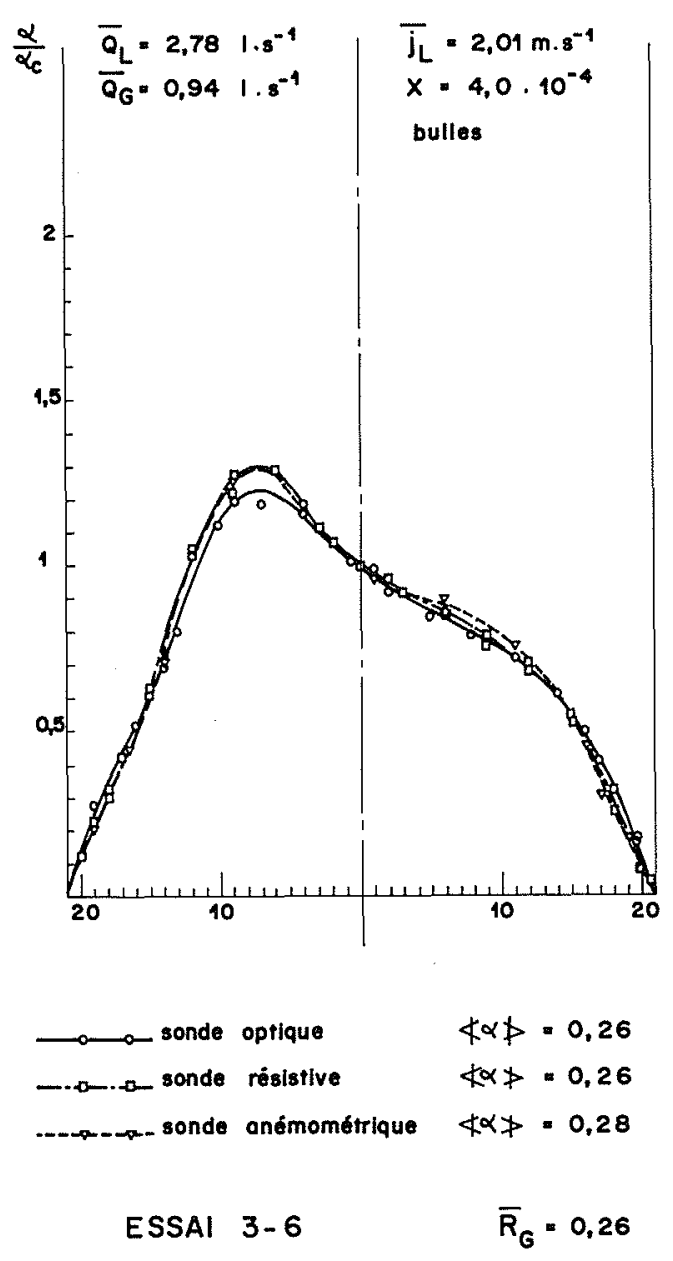

21 Comparaison des sondes locales.
Serizawa qui note des vitesses moyennes supérieures en moyenne aux vitesses mesurées par corrélation.

Les profils de vitesse obtenus sont présentés sur les figures 23 à 25 . Nous avons porté les vitesses moyennes mesurées à partir des histogrammes des temps de transit pour les bisondes optiques et résistives. Nous donnons également les profils correspondants de la vitesse locale du liquide mesurée par une sonde conique anémométrique à film chaud.

Nous comparons la vitesse apparente du gaz:

$$
\bar{i}_{i} \triangleq \bar{Q}_{i} / A
$$

où $A$ est l'aire de la section droite du tube avec la valeur globale déduite des mesures locales:

$$
\left\langle\bar{j}_{i j}(l)>=1 / A \int_{i} \alpha_{f i} \bar{V}_{i j} d A\right.
$$

Les résultats sont présentés dans le tableau 2 .

La comparaison n'est que très approximative car la précision des mesures est insuffisante. Des expériences complémentaires sont nécessaires.

Il semble cependant que l'on puisse estimer que la vitesse moyenne mesurée par une bisonde détectrice de phase soit la vitesse locale du gaz.

\subsection{Mesure de la surface spécifique d'un écoulement à bulles}

\section{a) Définition et intérêt de ce paramètre}

La surface spécifique est égale à l'aire cumulée des interfaces gaz-liquide par unité de volume. Elle s'exprime donc par l'inverse d'une unité de longueur.

La connaissance de cette grandeur est d'un intérêt évident pour tous les problèmes de transferts de chaleur et de masse. Ainsi, les travaux les plus avancés dans ce domaine sont ceux qui touchent au génie chimique où les problèmes de transferts de masse jouent un rôle important dans l'étude de la cinétique et du rendement des réactions chimiques. Signalons ainsi l'article de Sharma et Danckwerts [18] qui passe en revue diverses méthodes chimiques de mesure de la surface spécifique dans le cas d'écoulements diphasiques à bulles, à bouchons ou annulaires. Toutes ces méthodes reposent sur la mesure de l'absorption d'un gaz (généralement du $\mathrm{CO}_{2}$ ) par une solution aqueuse d'un absorbant (généralement, un hydroxyde alcalin, $\mathrm{NaOH}$ par exemple). L'ordre de grandeur des surfaces spécifiques obtenues pour un écoulement à bulles dispersées varie entre 1 et $10 \mathrm{~cm}^{2} \cdot \mathrm{cm}^{-3}$. 


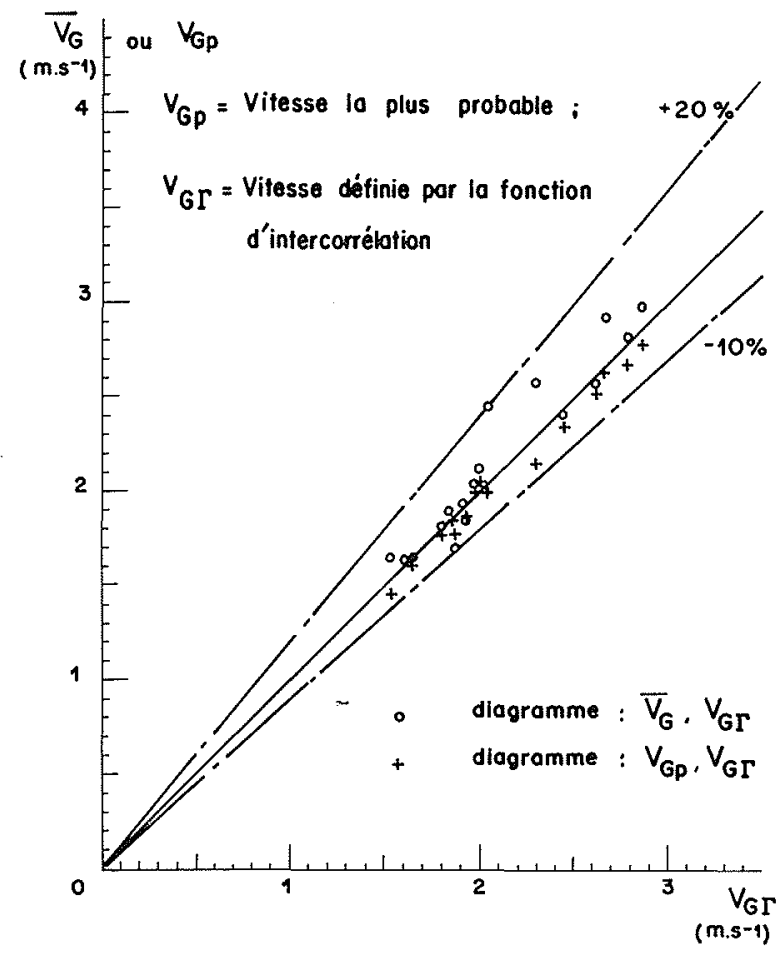

22/ Comparaison des mesures de vitesses des bulles par histogramme et par fonction de correlation.

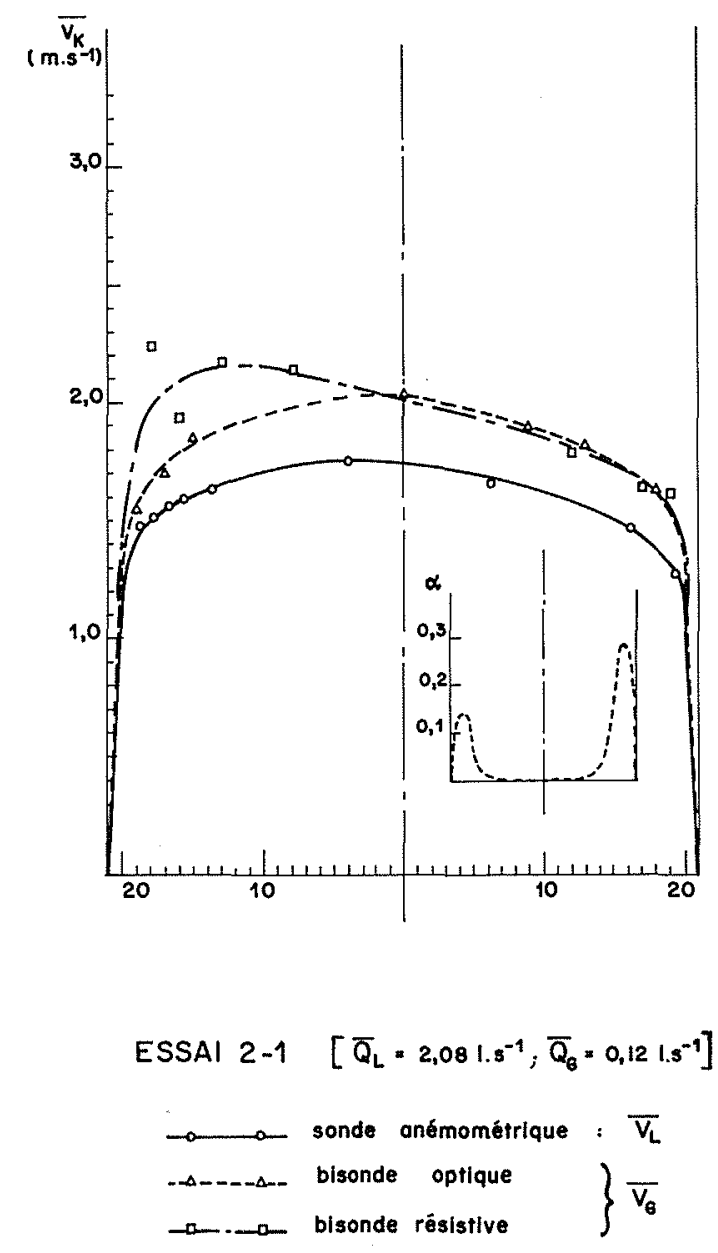

23/ Profils des vitesses d'un écoulement à bulles.

\begin{tabular}{|c|c|c|c|c|c|c|}
\hline $\mathrm{N}^{\circ}$ essai $\ldots \ldots \ldots \ldots \ldots$ & \multicolumn{2}{|c|}{$2-1$} & \multicolumn{2}{|c|}{$2-5$} & \multicolumn{2}{|c|}{$3-5$} \\
\hline $\bar{R}_{i_{i}} \ldots \ldots \ldots \ldots \ldots \ldots$ & \multicolumn{2}{|c|}{0,05} & \multicolumn{2}{|c|}{0,28} & \multicolumn{2}{|c|}{0,21} \\
\hline $\bar{j}_{i}\left(\mathrm{~m} \cdot \mathrm{s}^{-1}\right)$ & \multicolumn{2}{|c|}{0,085} & \multicolumn{2}{|c|}{0,51} & \multicolumn{2}{|c|}{0,51} \\
\hline bisonde ............ & opt. & res. & opt. & res. & opt. & res. \\
\hline$\left\langle\bar{j}_{g}(l)>\left(\mathrm{m} \cdot \mathrm{s}^{-1}\right) \ldots\right.$. & 0,069 & 0,074 & 0,57 & 0,59 & 0,58 & 0,59 \\
\hline$\frac{\leq{\overline{i_{G}}}(l)>-\bar{i}_{r}}{\bar{i}_{r_{i}}} \cdots \ldots \ldots$ & -0.19 & $-0,13$ & $+0,12$ & $+0,16$ & $+1.0,14$ & $+0,16$ \\
\hline
\end{tabular}




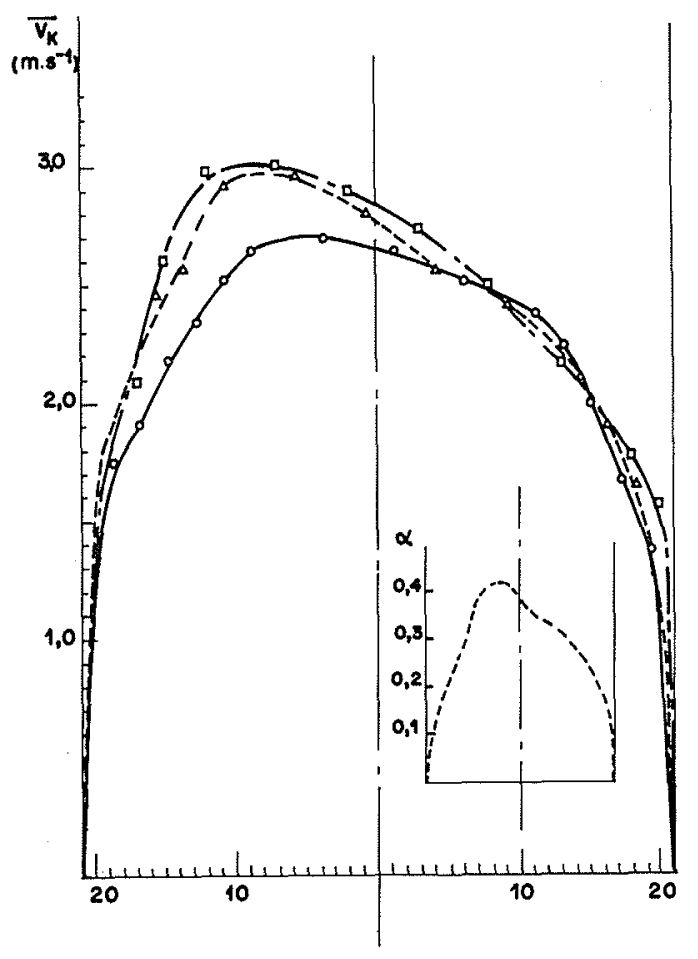

ESSAI $2-5\left[\bar{Q}_{L}=2,081.8^{-1}, \bar{Q}_{\theta}=0,711.8^{-1}\right]$

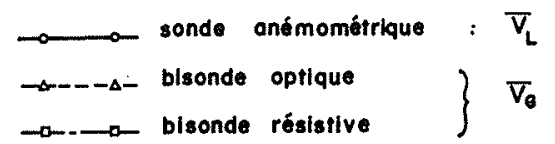

24/ Profils des vitesses d'un écoulement de transition.

Parmi les travaux récents, Kasturi et Stepanek [19, 20] se sont intéressés aux écoulements co-courants gazliquide dans un tube vertical de $6 \mathrm{~mm}$ de diamètre intérieur. Ils ont mesuré les surfaces spécifiques [19] et les coefficients de transfert de masse [20] pour différents régimes d'écoulement annulaire ou à bouchons.

L'un de nous [14] a montré que cette grandeur pouvait être également mesurée à l'aide d'une méthode physique (emploi de sondes) s'appuyant sur une analyse statistique de l'écoulement étudié.

\section{b) Détermination expérimentale}

Par définition, nous savons que :

$$
a=\frac{\sum_{i} a_{i}}{\mathfrak{V}}=\frac{\begin{array}{c}
\text { somme des aires des interfaces } i \\
\text { sans le volume } \vartheta
\end{array}}{\text { volume } \vartheta}
$$

En utilisant un résultat simple et général de la stéréologie [21], on peut montrer que [14] :

$$
a_{l}(r)=4\left[\overline{\nu(r)} / \overline{V_{G}(r)}\right]
$$
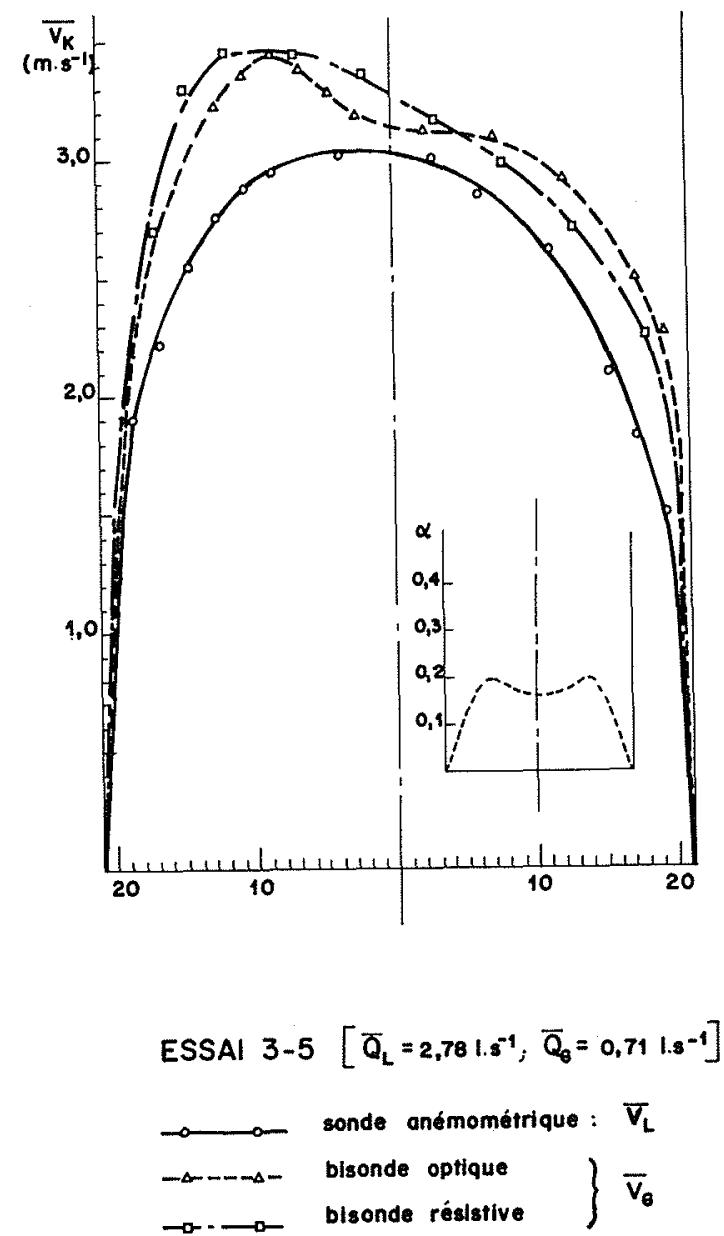

25/ Profils des vitesses d'un écoulement à bulles.

où $a_{l}(r)$ est la surface spécifique "locale », $\overline{\nu(r)}$ la fréquence moyenne de passage des bulles et $\overline{V_{G}(r)}$ la vitesse moyenne locale du gaz.

Si l'écoulement est axisymétrique, la surface spécifique globale est :

$$
a=8 / R^{2} \int_{0}^{l}\left[\overline{v(r)} / \bar{V}_{i}(r)\right] r d r
$$

Il est remarquable que, pour obtenir la surface spécifique, aucune donnée sur la granulométrie de l'écoulement ne soit nécessaire.

\section{c) Résultats}

L'application de la formule (12) a conduit aux résultats indiqués dans le tableau 3.

Les valeurs obtenues pour la surface spécifique sont en bon accord avec les résultats de Sharma et Danckwerts [18] issus de méthodes chimiques. En outre, un calcul approché effectué sur des photographies de l'écoulement a permis de retrouver l'ordre de grandeur des valeurs affichées. 


\begin{tabular}{|c|c|c|c|}
\hline \multicolumn{3}{|l|}{$\begin{array}{l}\text { Estimation de la surface } \\
\text { spécifique }\end{array}$} \\
\hline EssAI & $\bar{i}_{L}\left(\mathrm{~m} \cdot \mathrm{s}^{-1}\right)$ & $x$ & $a\left(\mathrm{~cm}^{2} \cdot \mathrm{cm}^{-8}\right)$ \\
\hline $2-1$ & 1,50 & $6,7 \cdot 10^{-5}$ & 1,5 \\
$2-5$ & 1,50 & $4,1 \cdot 10^{-4}$ & 3,1 \\
$3-5$ & 2,01 & $4,0 \cdot 10^{-4}$ & 3,0 \\
\hline
\end{tabular}

\section{Conclusions}

Le nouveau type de sonde optique utilisé dans cette étude s'est avéré un outil commode pour l'étude de la structure locale des écoulements diphasiques.

L'emploi d'une sonde simple permet la mesure du taux de présence d'une des phases dans un milieu diphasique où le liquide peut ne pas être conducteur de l'électricité.

L'emploi d'une sonde double, constituée de deux éléments sensibles, permet l'estimation de la vitesse locale de la phase gazeuse.

\section{Nomenclature}

$A$ aire des interfaces, aire de la section droite du tube;

a surface spécifique:

$D$ diamètre;

d distance:

$i$ angle;

j vitesse apparente;

$k$ numéro de bulles:

$N$ nombre de bulles;

$n$ indice de réfraction;

$Q$ débit volumique;

$R \quad$ taux de présence sur un segment, rayon de la conduite;

$r$ rayon;

$T$ temps de mesure;

v volume de contrôle:

$V$ vitesse;

$x$ abscisse; titre massique.

$\alpha \quad$ taux de présence local;

$\Gamma$ fonction d'intercorrélation;

$\theta$ temps de transit :

$\checkmark$ fréquence de passage des bulles;

$\tau$ temps.

\section{Indices}

$G \mathrm{gaz}$;

$i$ interface:

$L$ liquide; local ;

incident;

probable.

\section{Références}

[1] BOURÉ (J. A.). - Modeling methods in two-phase flow thermo-hydraulics. "Thermoidraulica dei fluidi bifase ". Comitato Nationale Energia Nucleare (1972) p. 233249.

[2] Miller (N.), Mitchie (R. E.). - The development of a universal probe for measurement of local voidage in liquid/gas two-phase flow systems. "Two-phase flow instrumentation", A.S.M.E., Le Tourneau (B.W.) and Bergles (A. E.), éd. (1969) p. 82-88.

[3] Miller (N.), Mitchie (R. E.). - Measurement of local voidage in liquid/gas two-phase flow systems using a universal probe. J. of the British Nuclear Energy Society $(1970)$ vol. $9, \mathrm{n}^{\circ} 2$, p. 94-100.

[4] Bell (R.), Boyce (B. E.), Collier (J. G.). - The structure of a submerged impinging gas jet. $J$. of the British Nuclear Energy Society (1972) vol. 11, $\mathrm{n}^{0}$ 2, p. 182-193.

[5] Kennedy (T. D. A.), Collier (J. G.). - The structure of an impinging gas jet submerged in a liquid. "Multiphase flow systems ». (1974), The Institution of Chemical Engineers Symposium, series $\mathrm{Nr} 38$, vol. II, paper J 4.

[6] Hivata (S.). - A study on the measurement of the local void fraction by the optical fibre glass probe. Bulletin of J.S.M.E., vol. $15, \mathrm{n}^{\circ} 88$, p. $1228-1235$.

[7] Powell (J. A.). - A simple two-fiber optical displacement sensor. Review of Scientific Instruments (1974) vol. 45, n" 2, p. 302-303

[8] Dayet. (F.), Delhaye (J. M.). - Sonde optique pour la mesure du taux de présence local en écoulement diphasique. Mesure, Régulation, Automatisme (aoûtseptembre 197I) p. 99-101.

[9] Delhaye (J. M.). - Local Measurements in two-phase flow. "Fluid dynamic measurements in the industrial and medical environments", vol. 1, Conference papers. Ed. by D. J. Cockrell, Leicester Univ. Press (1972) p. 191-200.

[10] Galaup (J. P.). - Sondes optiques. "Métrologie des écoulements diphasiques. Quelques procédés».C.E.A., R 4457 (1973) p. 79-89.

[11] Galaup (J. P.), Charlot (R.). - Mesure du taux de vide local à l'aide d'une sonde optique. $C E N-G$, note TT $\mathrm{n}^{0}-458$ (1974).

[12] Delhaye (J. M.), Galaud (J. P.). - Measurement of local void fraction in Freon 12 with a $0.1 \mathrm{~mm}$, optical fiber probe. European two-phase flow ineeting (3-7 June 1974) Harwell 
[13] Cette deuxième version est fabriquée par la Société Assistance Industrielle Dauphinoise (A.I.D.), 35, ch. de Halage, 38000 Grenoble.

[14] Galaup (J. P.). - "Contribution à l'étude des méthodes de mesure en écoulement diphasique", thèse de docteur-ingénieur. Université Scientifique et Médicale de Grenoble, Institut National Polytechnique de Grenoble (1975).

[15] Delhaye (J. M.). - " Contribution à l'étude des écoulements diphasiques eau-air et eau-vapeur ", thèse de Doctorat es Sciences physiques. Université de Grenoble, (1970).

[16] Delhaye (J. M.), Galaup (J. P.). - Hot-film anemometry in air-water flow. Fourth Biennial Symposium on Turbulence in Liquids, Univ. of Missouri-Rolla (1975).
[17] Serizawa (A.). - "Fluid dynamic characteristics of two-phase flow ». Institute of Atomic Energy, Kyoto University (1974).

[18] Sharma (M. M.), Danckwerts (P. V.). - Chemical methods of measuring interfacial area and mass transfer coefficients in two-fluid systems. British Chemical Engineering, vol. 15, no 4 (1970) p. 522-528.

[19] Kasturi (G), Stepanek (J. B.). - Two-phase flow. III : Interfacial area in co-current gas-liquid flow. Chemical Engineering Science, vol. 29, (1974) p. 713-719.

[20] Kasturi (G.), Stepanek (J. B.). - Two-phase flow. IV : Gas and liquid side mass transfer coefficient. Chemical Engineering Science, vol. 29 (1974) p. 1849-1856.

[21] UnderwoOd (E. E.). - "Quantitative stereology". Addison-Wesley Publishing Company (1970). 